\title{
A METHOD FOR INCREASING THE EFFICIENCY OF A WIND TURBINE
}

\author{
Yershina A.K., Sakipova S.E. \\ 1Kazakh National Women's Teacher Training University, Almaty, Kazakhstan, 123ainakul.yershina@gmail.com \\ 2E.A. Buketov Karaganda University, Karaganda, Kazakhstan, sesaule@mail.ru
}

\begin{abstract}
The article discusses the possibilities of improving the energy efficiency of wind turbines. A brief analysis of the current state of development of alternative energy, in particular wind energy in Kazakhstan, is considered. The computational study has shown that an increase in the power of a wind-power plant is possible due to the use of a system of simultaneously rotating rotors. A brief description of Bidarrieus-2 wind turbine construction, which can significantly increase the power removed from the wind flow, is given. The possibility of further increasing the efficiency of the Bidarrieus-2 wind turbine by means of a concentrator is discussed. The unique method for the speed controlling of the wind flow with the help of sliding flaps of the guide concentrator in order to improve the performance of the wind turbine at high wind speed is proposed first time.
\end{abstract}

Keywords: wind power, vertical-axial wind turbine, multy-rotor unit, Bidarrieus-2, wind energy utilization factor.

\section{Introduction}

The problems of warming and climate change caused by ever-increasing harmful emissions into the atmosphere have led to a widespread transition from hydrogen energy to the development and implementation of technologies for converting renewable energy sources (RES) [1-5]. Kazakhstan has also made voluntary commitments to reduce harmful carbon emissions to zero by 2050. Our country possesses significant renewable energy resources, which include hydropower, wind and solar energy. The number of renewable energy projects being implemented annually increases.

The highest growth rates of electricity from renewable energy sources excluding large hydroelectric power plants in Kazakhstan were observed in 2011-2016. [1]. Although the growth rates of RES facilities slightly decreased, but continue to grow: by the end of 2017, 58 RES facilities with an installed capacity of 342.3 MW were commissioned, and by the end of 2018, 67 RES facilities with a total installed capacity of 531 MW were in operation, which amounted to an increase in the number by $19 \%$ and power by $55 \%$ [3]. As of July 1, 2019, 75 renewable energy facilities with a total installed capacity of 683.6 MW were commissioned, of which 16 wind power plants (232 MW), 23 solar photovoltaic plants (250 MW), 34 hydroelectric power plants (200.3 MW) and 2 bioelectric power plants (1.3 MW).

Practice shows that wind energy is the most preferable among the fairly actively introduced renewable energy facilities. Now wind power is one of the fastest-growing renewable energy technologies. Usage is on the rise worldwide, in part because costs are falling.

Installed capacity, MW

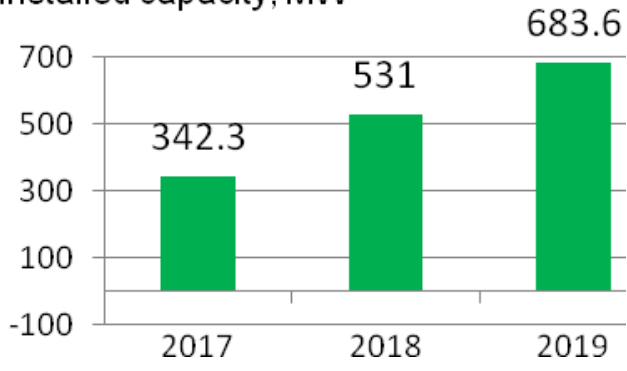

Fig.1. Dynamics of renewable energy facilities in Kazakhstan.

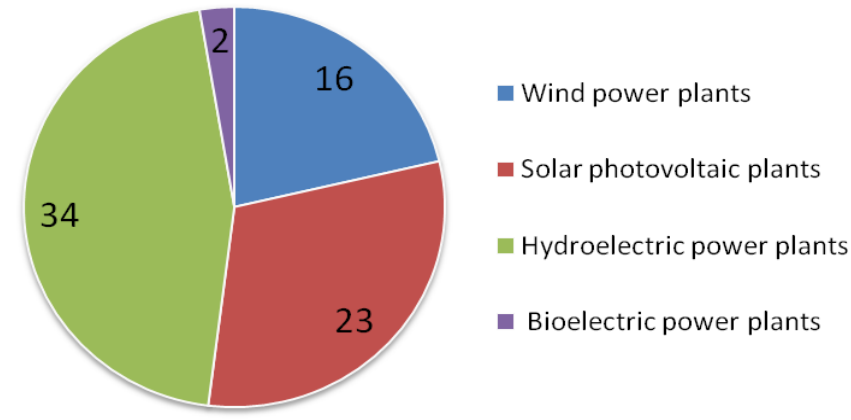

Fig.2. Types of renewable energy facilities in Kazakhstan. 
According to [2] the production of wind electricity in 2016 accounted for a $6 \%$ of the electricity generated by RES. Wind energy not only in Kazakhstan is actually becoming the basis for both a strategy to reduce greenhouse gas emissions and a rejection of the use of fossil and nuclear energy. According to the official statistical report of the World Wind Energy Association Global Status Report 2020, the total capacity of installed wind turbines in the world reached $651 \mathrm{GW}$ by the end of 2019 [3].

Despite the active development of the RES sector in Kazakhstan, there are still problems [4-5]. It should be noted that the emergence and rapid spread of COVID-19, which began in late 2019, turned into a global pandemic by early 2020, leading to a global health and economic crisis. It has also impacted the energy sector around the world. Many parts of the world have strong wind speeds, but the best locations for generating wind power are sometimes remote ones. It is quite possible to solve some problems, for example, training qualified engineering personnel for servicing wind energy facilities, creating a new pricing model in the field of electricity production, etc. But to solve a number of problems, significant capital investments are required from the state and large energy corporations: these include insufficient development of domestic capacities of wind power plants (WPPs), the impossibility of integrating the energy generated by individual WPPs into the general power supply system, and the lack of sufficiently effective technologies for its storage (accumulation). Let us consider some aspects of increasing the efficiency of wind turbines.

\section{Energy efficiency of a wind turbine and multy-rotary wind units}

It is well known that in the development and creation of any machine an important and main characteristic of the usefulness of its use is the value of the coefficient of energy conversation efficiency [68]. During its operation, it characterizes the ratio of the benefits of using this machine to the required costs: cost, operating costs, operating life, payback, etc. This also applies to the operation of wind power units. Here, the coefficient of energy conversation efficiency of the wind unit is determined by the wind energy utilization factor $\xi$ (WEUF). The higher the $\xi$ value means the more effective the economic and commercial value of the wind turbine. Obviously, the larger the area of the wind wheel swept by the wind, the greater the value of the WEUF should be. Over the past few decades, the scale of wind turbines has increased significantly, this has led to a decrease in the cost of electricity generated [2, 9]. It is necessary to increase the size of the wind turbine in order to reduce the normalized cost of energy. But increasing the power of the turbine by simply increasing the direct dimensions of the blades in practice requires large expenditures for their manufacture, installation and operation. In addition, it is impossible to infinitely increase the size of the blades. Therefore, the researchers propose as an alternative method for solving the problem of increasing the WEUF - to use a system of simultaneously rotating several rotors (two or more), instead of one.

The idea of a multi-rotor installation is not new. At the beginning of 2016, a wind turbine with a capacity of $900 \mathrm{~kW}$ was erected at a test site located near Roskilde (Denmark) as part of a research and development project, Fig.3 [9]. This wind turbine, as part of a wind power plant (WPP), was equipped with four V-29-225kW wind turbines with a controlled pitch and a rotor diameter of $29 \mathrm{~m}$. After 2.5-years tests, Vestas company dismantled an experimental 4-rotor wind turbine. The processes of interaction between the wind turbine blades and their effect on the turbine aerodynamics were studied. The influence of multi-rotor speed on the noise level and other wind turbine characteristics were also investigated. The tests carried out by the Danish company confirmed the improvement in the quality of the wind turbine. Preliminary results of studies on a 900-kilowatt wind turbine showed a $1.5 \%$ increase in power in relation to annual energy production, compared with a separately rotating rotor [9]. It was found that the 4-rotor version of the installation has the property of a faster leveling of the vortex wake behind the wind generator, which in a real situation means the possibility of installing the turbines at a closer distance relative to each other. Probably it's the result of the overlapping of the perimeters of the rotors, which is equal in area to two perimeters of a single-rotor turbine for the same swept area.

The relevance of this direction of innovation and technology modernization for vertical-axis wind turbines is confirmed by a number of publications [10 - 14]. In [10], the authors studied the dynamics of the vortex flow on the basis of numerical simulations in order to compare the flow characteristics in the wake of a multi-rotor wind turbine with the characteristics of a single rotor turbine. In numerical experiments [10], it was found that the wake of a multi-rotor turbine recovers faster at short distances downwind. In [11], the aerodynamics of a double vertical-axial wind turbine with a deflector is studied. The performance of the system was quantified by aerodynamic tests followed by 3D fluid dynamics simulations. Thus, to meet the 
need for increasing power, the creation of multi-rotor wind generators becomes a promising alternative to massive single-rotor wind generators.

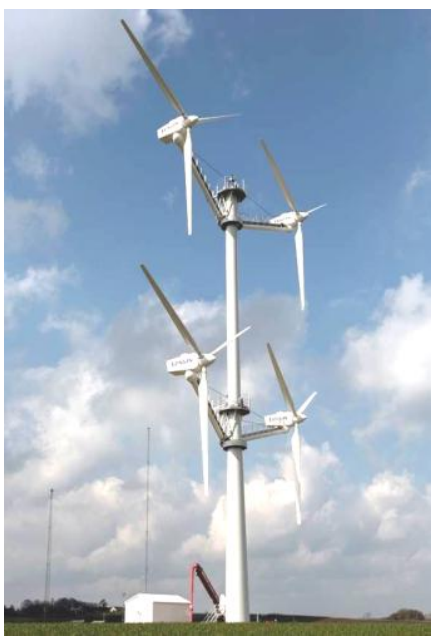

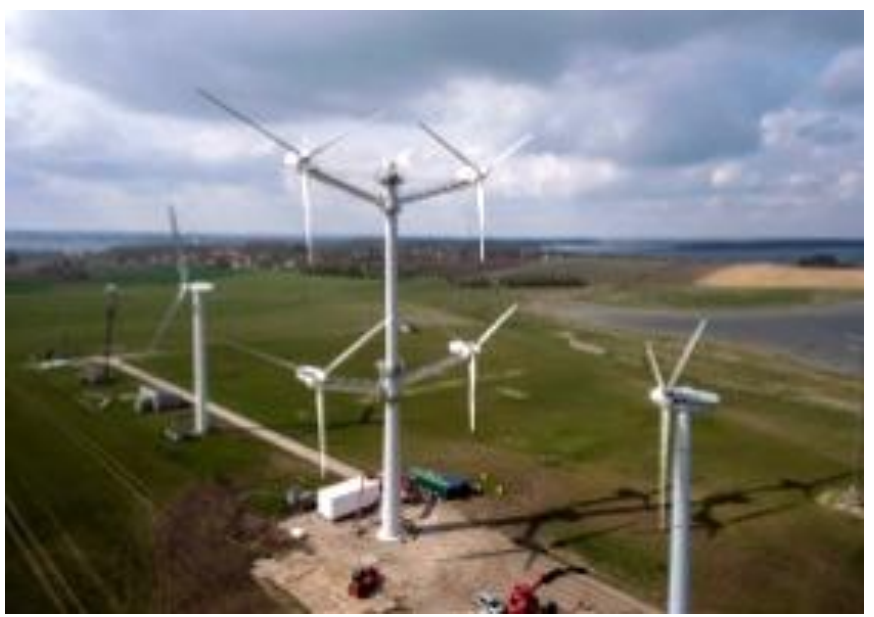

b

Fig.3. «Vestas» multi-rotor wind farm:

a) main view of a 4-rotor wind turbine; b) experimental wind turbine test farm [9].

In Kazakhstan, combined vertical-axial devices with two coaxially located rotation shafts were first developed more than 20 years ago by a group of scientists $[7,8]$. As a result of calculations and tests, three versions of two-rotor wind turbines have been proposed: Darrieus type, Bidarrieus-1, Bidarrieus-2. The most effective is the third version of the Bidarrieus-2 wind turbine, the fundamental feature of which is that its swing is not rectilinear, but L-shaped in the form of two half swings connected to each other on one of the shafts $[7,15]$. The uniqueness of this design is that the power taken by two DC electric generators is summed up. For centering, coaxially mounted rotation shafts are separated from each other by support bearings, which makes it possible for their independent rotation: both coordinated in the same direction, and opposite. The rotor blades can be made in the form of NASA symmetrical wing profiles. This design, together with the HBI rotor, allows you to get almost 2 times more streamlined area than a conventional Darrieus windmill. So, the amount of wind energy used by wind turbines with HBI rotor doubles [15].

Laboratory tests and calculations have shown that such a constructive solution significantly increases the value of WEUC, and, accordingly, the practical efficiency and commercial viability of wind turbines [16]. The tests of the laboratory sample and calculations have shown that such a constructive solution significantly increases the value of WEUC, and, accordingly, the practical efficiency and commercial feasibility of wind turbines [7]. The autonomy of the shaft rotation of the carousel type wind turbine allowing energy to transfer each working blade to two different generators, thus increasing the total energy received, i.e. increase the value of the $\xi$ coefficient.

The developed semi-industrial Bidarrieus wind turbine with a $5 \mathrm{~kW}$ HBI-rotor showed the need for its placement at a certain height $[8,16]$. Outside the surface boundary layer where wind speeds are 2-2.5 times higher than the average wind speed in the surface boundary layer. Due to the cubic dependence of the wind turbine power on wind speed, the location of the wind turbine outside the surface boundary layer (20-30 m) will lead to a several-fold increase in the power of the wind turbine. The torque and power are determined by loading the pulley with calibrated loads mechanically by the friction method [6]. With varying degrees of loading the wind turbine, the number of shaft speed is measured using an electronic tachometer. The speed of the wind turbine is determined by the number of shaft. The torque $\mathrm{M}$ created by the wind turbine is determined by the well-known correlation of the theory of similarity:

$$
M=m \cdot \frac{\rho V^{2}}{2} \cdot \mathrm{S} \cdot \mathrm{R},
$$

where $m$ is the dimensionless coefficient of torque, $\frac{\rho V^{2}}{2}$ is the dynamic pressure of the incoming flow, $\mathrm{U}$ is the flow velocity (determined by two calibrated anemometers), $\rho$ is the air density, $\mathrm{S}$ is the swept area, $\mathrm{R}$ is the radius of the turbine and $\mathrm{H}$ is its height. 
From the calculated values of the torque and speed of the wind turbine, the values of the wind energy utilization coefficients are determined:

$$
\xi=m \cdot \chi,
$$

where $\chi$ is tip speed ratio.

Then, the dependence of $\xi$ on speed for different wind turbine models is built and a comparative analysis of wind turbines is carried out.

\section{A method further increasing the efficiency of two-rotor wind turbine using a concentrator.}

For the purpose of increase in wind speed operating in a windmill the concentrator of wind energy is offered. In Fig. 4 the design of Bidarrieus-2 with the wind power concentrator is shown in axonometric. As shown in figure 5 the motionless concentrator represents a system of convergent guides located in a uniform fantail around the windmills. To increase the velocity of wind acting on the wind power unit, a concentrator of wind energy is proposed.

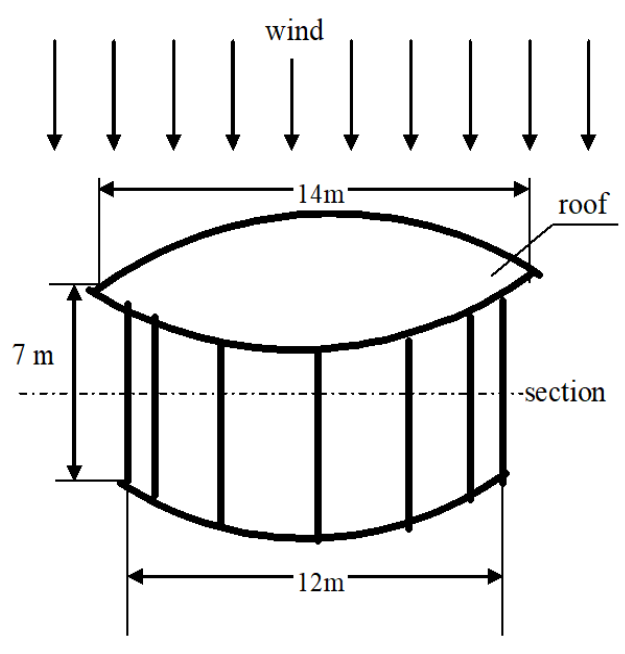

Fig. 4. Wind power concentrator design.
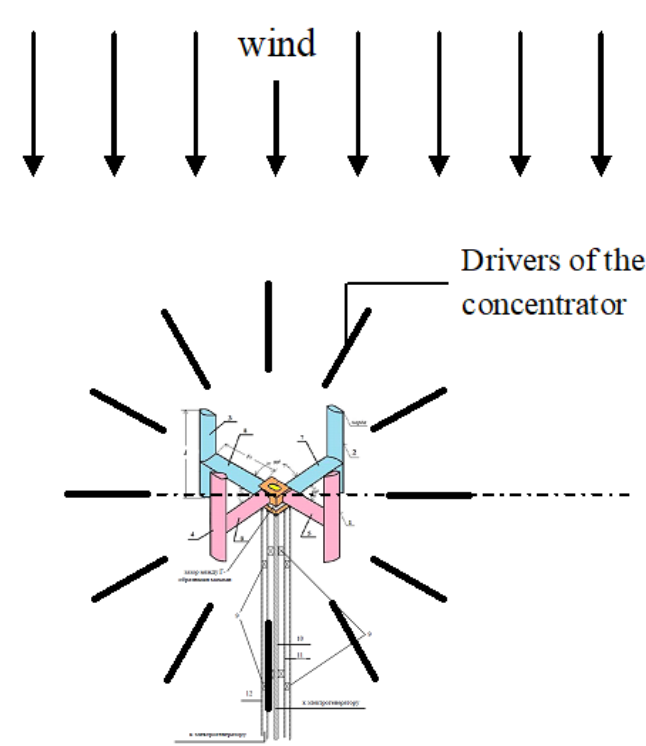

Fig.5. General view of Bidarrieus-2 with wind power concentrators.

A concentrator is a system of converging guides positioned as a uniform fan around wind power units. The sizes of guiding concentrators in this case allow increase more than twice the velocity of wind flow acting on the unit at any direction of wind. For six-meter Bidarrieus-2 the diameter of total area taking into account the concentrator is equal to $14 \mathrm{~m}$. The total height is $7-8$ meters. All this construction is protected by a roof with a small cone angle [15]. Diameter of this roof is at 1 and 2 meters more than diameter of whole construction. Let's enter acceleration factor of a wind stream in concentrator as the relation

$$
\varkappa=\frac{\mathrm{U}_{1}}{\mathrm{U}_{2}},
$$

where:

$\mathrm{U}_{1}$ - speed at the entrance of the wind stream in concentrator,

$\mathrm{U}_{2}$ - exit speed from concentrator.

The guides of concentrator consist of two flaps which can open as a book. The material of guides must be from dielectrics and must conduct heat well. To the internal side of each flap, tans are pasted which heat the guides and in winter protect the whole construction from wet snow-drift. It's an effective method of additional regulation of speed of a wind stream by means of moving apart flaps concentrator guides. Devices for directing can perform one more function. You can reveal them and regulate the wind speed, which is acting on the wind turbine. The movement of the leaflets is controlled by the axes of rotation like on the "book binding". 


\section{Conclusion.}

Two-rotory Bidarrieus-2 wind turbine was proposed first time in Kazakhstan. A vertical-axis wind turbine is the most promising converter of wind energy, especially in conditions of rapid changes in wind speed and direction. The relevance and necessity of developing this wind turbine is also justified by the fact that its use for generating electricity is beneficial and economical for decentralized regions and in remote places. In addition, the double-rotor wind turbine of the carousel type Bidarrieus-2 has a low rotation speed. This allows it to work at high wind speeds, and the service life is practically unlimited. All this makes it important and necessary to carry out full-scale tests of these WPPs in order to ensure their industrial production in the future.

\section{REFERENCES}

1 Development of green energy in Kazakhstan. 19.09.2018. Available at: https://eenergy.media/2018 / 09/19 / razvitie-zelyonoj-energetiki-v-kazahstane /

2 Wind Energy Data. International Renewable Energy Agency (IRENA). Available at: https://irena.org/wind

3 Renewable Energy 2020. Statistical Global Status Report. Available at: https://www.ren21.net/reports/globalstatusreport

4 Mukhamadiev IR, et al. Multi-criteria spatial decision-making system to support the development of RES in Kazakhstan. IEEE Access, 2019, Vol. 7, pp. 122275 - 122288.

5 Zhunusova G. Zh. et al. Renewable energy in Kazakhstan: challenges and prospects. International Energy Journal. 2020, pp. $311-324$.

6 Bezrukikh P.P. Ispol'zovanie energii vetra.( Wind power use). Moscow, 2008, 196 p. [in Russian]

7 Yershin Sh., Yershina A.K About High efficiency of two-rotor wind power unit Bidarrieus-2. Eurasian Physical Technical Journal. 2019, No. 1(31), pp. 82 - 87. DOI 10.31489/2019No1/82-87

8 Yershina A.K., Sakipova S.E., Manatbayev R.K. Some desing featurs of the carousel type wind turbine Bidarrieus. Eurasian Physical Technical Journal. 2019, No. 2(32), pp. 63 - 67. DOI: 10.31489 / 2019 No2 / 63-67.

9 Eize de Vries. Vestas tests four-rotor concept turbine. 04/20/2016. Available at: www.windpowermonthly.com /article/ 1391775/ exclusive-vestas-tests-four-rotor-concept-turbine

10 Mosfequr Rahman, et al. Numerical and Experimental Investigations on Vertical Axis Wind Turbines of Different Models. Open Access Library Journal. 2017, Volume 4, e3273. DOI: 10.4236/oalib.1103273

11 Yichen Jiang, Peidong Zhao, Thorsten Stoesser, Kun Wang, Li Zou. Experimental and numerical study of dual vertical-axis wind turbines with a deflector. Energy Conversion and Control. 2020, Vol. 209, pp. 112588. https://doi.org/10.1016/ j.enconman.2020.112588.

12 Ahmedov A., Ebrahimi K. M. Numerical Modelling of an H-type Darrieus Wind Turbine Performance under Turbulent Wind. American Journal of Energy Research. 2017, Vol.5(3), pp. 63 - 78. DOI: 10.12691/ajer-5-3-1

13 Longhuan Du, Ingram, Grant Dominy R. A review of H-Darrieus wind turbine aerodynamic research. Journal of Mechanical Engineering Science. 2019, Vol. 233, Issue (23-24), pp.7590-7616.

14 Kayan V.P., Kochin V.A., Lebid O.G. Studying the Performance of Vertical Axis Wind Turbine Models with Blade Control Mechanism. Intern. Journal of Fluid Mechanics Research, 2009, Vol.36, Issue 2, pp. 154 - 165.

15 Yershina A.K., Yershin Sh., Yershin Ch.Sh. Manatbaev R.K. Wind turbine. Patent KZ. Reg. No. 2016/0337.1. Bul. No. 15. Publ. 04/11/2016.

16 Yershina A.K., Manatbayev R.K., Sakipova S.E., Kalasov N.B. Improving the efficiency of wind turbine. Proceeding of the $2^{\text {nd }}$ Intern. Scient. Conf. “Alternative Energy Sources, Materials \& Technologies (AESMT'19), Sofia, Bulgaria. 2019, Vol. 1, pp. $89-90$. 